\section{Role of fine needle aspiration cytology}

Joan Lamb and her colleagues are to be congratulated on their timely publication on the role of fine needle aspiration cytology in breast cancer screening.' There are, however, some points which require clarification as they have a bearing on the interpretation of the authors' results in relation to the provision of a similar service in other districts where breast cancer screening is to be undertaken.

What was the overall size of the screened population that generated 562 aspirates and exactly what criteria were used to select the patients from the screened group who were deemed suitable for fine needle aspiration? Did all the selected patients have a palpable lesion, or were some of the aspiration procedures mammographically or radiologically "targeted", or even performed "blindly"?

It may be inferred from the paper that the aspirates were not performed by the pathologist who was to report on the specimen. We feel that this is an important point. It is certainly no accident that in Scandinavia where fine needle aspiration cytology is most widely practised, aspiration is generally performed by the cytopathologist.

At this hospital aspiration cytology has been performed on patients presenting at the breast clinic with a palpable mass since September 1986. Most aspirates are performed by a pathologist attending the clinic who personally stains and reports the specimen within the clinic. The pathologist benefits from a clinical impression of the nature of the lesion, and the speed of the procedure means that a scanty or inadequate aspirate can be immediately repeated for a second, or on rare occasions, even a third time to gain a diagnostic sample. The use of an ethyl chloride spray on the skin before aspiration makes multiple attempts more acceptable to the patient. These advantages are reflected in the overall results that we obtained. One hundred and fifty aspirations were performed over nine months, and of these, 109 were carried out by the same pathologist. A definite cytological diagnosis was possible in all 109 patients aspirated, and we believe this to be a direct consequence of the pathologist performing his or her own aspirations. In those cases in which histological results were subsequently available $22 \%$ were confirmed as benign, $66 \%$ were confirmed as malignant, $4 \%$ diagnosed as suspicious were found to be malignant and $8 \%$ diagnosed as benign were found to be malignant (aspirates negative on review). There were no false positive results but one case reported as suspicious proved histologically to be a benign fibroepithelial neoplasm. The benefits of the pathologist as aspirator, particularly with respect to the sensitivity of the procedure and the reduction in numbers of suspicious and inadequate samples cannot be emphasised too much. While there are obviously important differences between the role of fine needle aspiration in screening and in the different population of women presenting with a palpable mass, we believe that the principle of pathologist as aspirator should be extended into the screening process and this should help to keep inadequate samples or "suspicious" diagnoses to a minimum. The psychological morbidity generated by a screening programme is not to be underestimated and the facility of giving the women a rapid diagnosis at the stage of initial specialist assessment must surely be of benefit.

CS CHAMP,
CH MASON,
SB COGHIL,,
SJ POWIS
Department of Pathology,
Northampton General Hospital,
Cliftonville,
Northampton NN1 5BD

Reference

1 Lamb I, Anderson TJ, Dixon MJ, Levick PA. Role of fine needle aspiration cytology in breast cancer screening. J Clin Pathol 1987; 40:105-9.

\section{Drs Lamb and Anderson comment:}

As commented on in the results and discussion sections of our paper, we undertook a retrospective non-consecutive study of fine needle aspiration cytology in a breast cancer screening programme. The details of the population and methods of selection of women for further investigation have been given in a publication describing the protocol.'

None of the cases was radiologically targetted for aspiration, although this is now part of our procedures.

With regard to the aspirator, we think the most important factors are motivation, aptitude, and ability to make good smears. Local situations and availability of personnel will vary, and the degree of collaboration between the different disciplines of surgery, radiology, and pathology will affect the quality of service provided.

JOAN LAMB TJ ANDERSON
Department of Pathotgy, University Medical School,

Teviot Pace,

Edinburgh EH8 \&AG

Reference

言

1 Roberts MM, Alexander F, Anderson TJ, st al. The Edinburgh randomised trial of scresing for breast cancer: description of method $\mathrm{BBr} J$ Cancer 1984;50:1-6.

\section{Mucinous thyroid carcinomas}

I read with interest the article by Rigaud Bogomoletz about mucin secreting cand mucinous primary thyroid carcinomas. "The authors concluded that, "carbohydrates contained in thyroglobulin and colloid could well be responsible for the apparent 'positive' staining obtained with the conventional histochemical methods for mucins, and $\$$ his could apply to thyroid tumours of both follicular and C cell origin."

Recently, is has been shown that thyofid follicles with acid mucins mostly composed of, and or related to, $\mathrm{C}$ cell conglomerattes (sometimes true mucinous $C$ cell complexes) exist. It was also shown that acid mucip is present in the cytoplasm of $C$ cells these follicles. ${ }^{2}$ These secondary type ${ }^{\circ}$ of thyroid follicles are anatomically relae to the mucin containing $C$ cells or ultimot chial solid cell nests of the thyroid. ${ }^{23}$ According to the conclusions of Rigaudând Bogomoletz, 'it would have been expecte. to find acidic mucins in the colloid of thyeid follicles not related to solid cell nests, \$̧ut acid mucosubstances were not present inthe many control sections far from the ultimobranchial nests derived from the gutt. ${ }^{2}$

I think that it is plausible to assume that the histogenesis of some mucin-producing thyroid tumours, especially meduhary carcinoma ${ }^{4}$ could be linked to the "ultimobranchial" thyroid follicles with @્cid mucins. ${ }^{2}$

HR HARQCH

Pathology Laboratery,

Department of Radiotherapy and Oncolegy, University Central Hosptital, Haarmaninkaou 4 00290 Helsin $\breve{k i j} 29$ Fin ßnd

References $\omega$

1 Rigaud C, Bogomoletz WV. "Mucin secret庐g" and "mucinous" primary thyroid carcinomas: pitfalls in mucin histochemistry applied to thyroid tumours. J Clin Pathol 1987;40:89 5 . 
2 Harach HR. Thyroid follicles with acid mucins in man. A second kind of follicles? Cell Tissue Res 1985;242:211-15.

3 Harach HR. A study on the relationship between solid cell nests and mucoepidermoid carcinoma of the thyroid. Histopathology 1985;9:195-207.

4 Zaatari GS, Saigo PE, Huvos AG. Mucin production in medullary carcinoma of the thyroid. Arch Pathol Lab Med 1983;107:70-4.

\section{Drs Rigaud and Bogomoletz comment:}

We thank Dr Harach for his comments on our recent paper. Firstly, we would like to emphasise that the aim of our study was to draw attention to pitfalls in mucin histochemistry when applied to thryoid tumours, in terms of methodology and interpretation. We only raised the possibility that "positive" staining, obtained with conventional histochemical methods for mucins, may be due to naturally occurring carbohydrates (from thyroglubulin or colloid). We did not refute the alternative that genuine mucin production could also take place in some thyroid neoplasms, through dual differentiation for instance. Obviously, solid cell nests derived from the ultimobranchial body could account for still another mechanism of true mucin secretion in thyroid tissue, as suggested by Dr Harach in his letter.

Secondly, we never claimed in our conclusions, as implied by Dr Harach, that positive staining of either genuine acidic mucins or thyroglobulin/colloid carbohydrates (containing sialic acid and sulphate radicals) is the rule in all thyroid tumours.

In our opinion, utmost caution is necessary in the interpretation of all primary thyroid neoplasms showing apparent "mucin secretion".

C RIGAUD, WV BOGOMOLETZ Institut Lean Godinot, Boite Postale 171, 51056 Reims, France

\section{Use of microcomputers to facilitate costing in} pathology laboratories

I congratulate Stilwell and Woodford on their recent article on microcomputer software. ${ }^{\prime}$ A most erudite and exhaustive procedure was described, providing a cornucopia of data which, while definitive in scope, was limited in its practical application. Its very comprehensiveness would necessitate considerable effort to start and even more to maintain.
Estimates of staff time particularly points II and III, ". . . . . how long and how much of his or her time spent on general duties," and "..... how much of his or her time spent on supervisory work on ..... tests, or at specified work stations," are not only inherently difficult to measure, but more importantly, vary each day according to demands and according to each individual's ability.

While I applaud the concept, some considerable simplification would be needed for actual use. I would respectfully suggest that the authors consider alternatives-for example, the CATER(chemical administrative time equipment reporting) system described by Stenton et al. ${ }^{2}$

To illustrate the difference between academia and the real world, I would direct your attention to the authors' statement on page 823, "Experience of the Canadian workload system is that 35 units per hour is a reasonable expectation." My experience in a routine hospital laboratory is, I am sure, not unique; recent information shows: microbiology $=84$ units activity per paid hour; haematology $=64$ units activity per paid hour; biochemistry $=50$ units activity per paid hour; histopathology $=57$ units activity per paid hour. My understanding of the Canadian workload system is that 44 minutes/paid hour should be taken as a target of $100 \%$ efficiency. ${ }^{3}$

Given a staffing of 35 units per hour I am sure that such a costing exercise would "seem" practical if somewhat expensive.

The unconstrained pursuit of accuracy is never cost effective and one doubts the need for the decimal place in realistic management.

CB BROWNHILL Pathology Service, Nevill Hall Hospital, Abergavenny, Gwent, Wales

\section{References}

1 Stillwell JA, Woodford FP. Microcomputer software to facilitate costing in pathology laboratories. J Clin Pathol 1987;40:817-25.

2 Stenton P, Hyde K, Hoctor D. Pathology costing-Cater versus Körner.IMLS Gazette 1987;31:281-4.

3 Canadian workload measurement systemlaboratory Ottawa: Department of Supply and Services, 1982:4-2301-506.

\section{Mr Stilwell and Dr Woodford comment:}

We agree with Mr Brownhill that staff time for each laboratory test is difficult to measure and that it varies from day to day and week to week. Precisely this point was made in introducing our concept of broadly estimating the proportion-not the absolute measure-of time, on average, which each staff member devotes (a) to work which does not produce tests results and (b) to each kind of workstation over a lengthy period-for example, a year. It was never the intention to divide up the staff member's time exactly during a particular study period, which would inevitably be unrepresentative.

Several experienced laboratory directors, which we do not pretend to be, have convinced us that this proportional estimation is not difficult to make and is in fact the only way to compensate, in costing, for the fact that staff members do move from test to test and that staff of different grades and salaries perform some of the same tests.

Once the proportions of staff time (and associated actual staff cost) have been assigned to the different workstations-that is, groups of tests - the costs can be very easily redistributed over individual tests at those workstations in ratios based on some independent estimate of the average time required for each, such as Canadian workload units.

We find the CATER system more of a complication than a simplification. Firstly, its inventors incorporate into the CATER unit, which seems to be meant primarily as a manpower factor, four other elements of cost (chemicals, administration, equipment, reporting). Secondly, they assign a seemingly arbitrary number of CATER units to each type of test and then calculate the unit's cost value by dividing the total number of units into the total staff costs, irrespective of whether all the staff is engaged in test production. Our faith in the system is not enhanced by some curious discrepancies in the paper cited': the computer printout in table 1 shows the CATER unit for full blood count to be 150 , but table 2 shows it to be 3 . What seem to be faulty principles, are, of course, more important than simple errors.

Mr Brownhill's reference to the Canadian workload unit manual should, I think, read page 20 , not 2 , and his reading there has led him astray. The productivity of 44 units per paid hour calculated there refers to a hypothetical "sample laboratory", as is made clear on the previous page: "Suppose that a sample laboraory produced 498,000 laboratory units of work. ...". The Canadian workload unit manuals are careful not to prescribe an ideal productivity level, or even recommend working to a median productivity level. Our statement that 35 units per paid hour was a "reasonable" expectation was based in part on the results of DHSS studies of the Can- 